\title{
Multimorbidity prevalence and patterns and their associations with health literacy among chronic kidney disease patients
}

\author{
M. S. Gurgel do Amaral ${ }^{1}$ D . S. A. Reijneveld ${ }^{1}$ (D) - L. M. G. Meems ${ }^{2}$ (D) J. Almansa ${ }^{1}$ (D) . G. J. Navis ${ }^{3} \cdot$ A. F. de Winter $^{1}$ (D)
}

Received: 21 October 2021 / Accepted: 9 December 2021 / Published online: 5 January 2022

(c) The Author(s) 2022

\begin{abstract}
Background Health literacy is the ability to deal with information related to one's health. Patients with low health literacy have poor disease-management skills for chronic diseases, such as chronic kidney disease (CKD). This could influence the number and combination of their diseases.

Methods We included adult patients with CKD stages 1-5 from the Lifelines Study $(n=2,742)$. We assessed the association between low health literacy and the number and patterns of comorbidities, considering them globally and stratified by age and sex, using multinomial logistic regression and latent class analysis, respectively.

Results Low health literacy was associated with a higher number of comorbidities in the crude models, and after adjustment for age, sex, eGFR, smoking, and BMI. In the crude model, the OR for low health literacy increased from 1.71 (1.25-2.33) for two comorbidities to $2.71(2.00-3.68)$ for four comorbidities. In the fully-adjusted model, the associations remained significant with a maximum OR of $1.70(1.16-2.49)$ for four comorbidities. The patterns of multimorbidity were similar for low and adequate health literacy, overall and by sex, bur tended to be different for patients older than 65. Older patients with low health literacy had higher comorbidity prevalence and a relatively greater share of cardiovascular, psychiatric, and central nervous system diseases.
\end{abstract}

M. S. Gurgel do Amaral

m.silva.gurgel.do.amaral@umcg.nl

S. A. Reijneveld

s.a.reijneveld@umcg.nl

L. M. G. Meems

l.m.g.meems@umcg.nl

J. Almansa

j.almansa.ortiz@umcg.nl

G. J. Navis

g.j.navis@umcg.nl

A. F. de Winter

a.f.de.winter@umcg.nl

1 Department of Health Sciences, Community and Occupational Medicine, University of Groningen,

University Medical Center Groningen, Hanzeplein

1, Building 3217, room 617, 9713GZ Groningen,

The Netherlands

2 Department of Cardiology, University of Groningen, University Medical Center Groningen, Groningen, The Netherlands

3 Department of Nephrology, University of Groningen, University Medical Center Groningen, Groningen, The Netherlands 
Conclusions Among CKD patients, low health literacy is associated with more multimorbidity. Health literacy is not associated with patterns of multimorbidity in younger patients, but a difference was observed in older ones. Improving low health literacy could be an intervention efficient also in decreasing multimorbidity in CKD patients.

\section{Graphical abstract}

\section{Multimorbidity prevalence and patterns and their associations with health literacy among chronic kidney disease patients}

Population: Adults with CKD stages 1-5 $(n=2,742)$.

Main Measures: Health literacy and multimorbidity, defined as having CKD and at least one comorbidity. Comorbidities related to 11 disease domains, based on data of 40 physical and psychiatric diseases.

Conclusions: Low health literacy is associated with more multimorbidity. Health literacy is not associated with patterns of multimorbidity in younger patients, but it is slightly in older ones.

Table. Association between low health literacy and number of disease domains*

\begin{tabular}{|c|c|c|c|c|c|c|c|c|c|c|c|c|}
\hline \multirow{2}{*}{$\begin{array}{l}\text { Number of } \\
\text { domains }^{a}\end{array}$} & \multicolumn{3}{|c|}{ Crude model } & \multicolumn{3}{|c|}{$\begin{array}{c}\text { Adjusted for age and } \\
\text { sex }\end{array}$} & \multicolumn{3}{|c|}{$\begin{array}{l}\text { Adjusted for age, sex, } \\
\text { and eGFR }\end{array}$} & \multicolumn{3}{|c|}{$\begin{array}{l}\text { Adjusted for age, sex, } \\
\text { eGFR, smoking, and BMI }\end{array}$} \\
\hline & $\mathrm{OR}$ & $95 \% \mathrm{Cl}$ & $\mathrm{p}$ & $\overline{O R}$ & $95 \% \mathrm{Cl}$ & $p$ & $\overline{O R}$ & $95 \% \mathrm{Cl}$ & $\mathrm{p}$ & $\overline{Q R}$ & $95 \% \mathrm{Cl}$ & $p$ \\
\hline 1 & 1.25 & $0.90-1.72$ & 0.18 & 1.12 & $0.80-1.56$ & 0.52 & 1.13 & $0.81-1.58$ & 0.47 & 1.06 & $0.74-1.51$ & 0.74 \\
\hline 2 & 1.71 & $1.25-2.33$ & 0.001 & 1.42 & $1.02-1.98$ & 0.04 & 1.47 & $1.05-2.05$ & 0.02 & 1.31 & $0.91-1.88$ & 0.14 \\
\hline 3 & 2.29 & $1.68-3.10$ & $<0.001$ & 1.76 & $1.25-2.46$ & 0.001 & 1.83 & $1.30-2.58$ & $<0.001$ & 1.59 & $1.10-2.31$ & 0.01 \\
\hline 4 or more & 2.71 & $2.00-3.68$ & $<0.001$ & 1.92 & $1.36-2.71$ & $<0.001$ & 2.00 & $1.41-2.83$ & $<0.001$ & 1.70 & 1.16-2.49 & 0.006 \\
\hline $\mathrm{n}$ & 2,742 & & & 2,742 & & & 2,742 & & & 2,715 & & \\
\hline
\end{tabular}

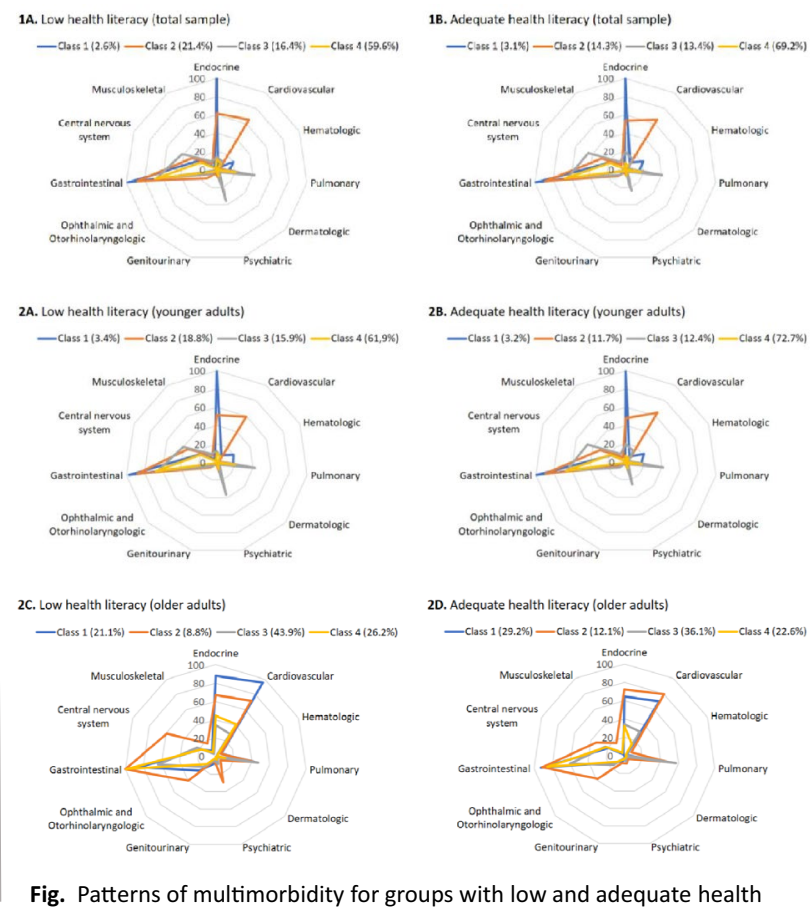

literacy, in the total sample (1A and $1 B)$, and stratified by age (2A-2D)

Keywords Health literacy $\cdot$ Multimorbidity $\cdot$ Patterns $\cdot$ Chronic kidney disease $\cdot$ Prevention

\section{Introduction}

Multimorbidity, the co-occurrence of two or more chronic diseases, is a condition that affects up to $95 \%$ of patients with chronic kidney disease (CKD) [1]. The term multimorbidity, however, does not specify the disease types and distribution, which are more informative of the impact these diseases have on people's health. Social determinants of health, such as health literacy [2], may help to determine the patterns of disease distribution in multimorbid patients with CKD, thereby supporting the development of strategies to approach these patients more efficiently.

Multimorbidity is a major challenge for patients with CKD [1], and a more comprehensive understanding of this phenomenon is needed to adapt healthcare systems to their needs [3]. Among CKD patients, multimorbidity is the single highest independent predictor of all-cause mortality [4], and it is an even more immediate concern than kidney function in the management of CKD, particularly during asymptomatic stages [5]. However, current healthcare systems and medical guidelines are generally focused on a single disease, resulting in fragmented and inefficient care, which fails to meet the challenges of multimorbidity [6]. To support the shift of healthcare systems to a more comprehensive model, we need to better understand the factors that determine the number and distribution of diseases in multimorbid patients [7].

Low health literacy is an important determinant of health due to its negative effects on chronic diseases [2], and it may be one of the determinants of multimorbidity among CKD patients. Health literacy is defined as the degree to which individuals have the capacity to obtain, process, and understand basic health information and services needed to make appropriate health decisions [8]. Low health literacy is associated with CKD development and complications $[9,10]$, mainly because individuals with low health literacy lack the self-management skills required for adequate CKD control $[2,11]$. This lack of skills may be especially problematic when the patient presents multimorbidity, since the co-occurrence of other diseases adds to the burden of CKD, making it more difficult to manage [12]. 
Low health literacy may predict not only the presence of multimorbidity but also its patterns, i.e., the combination of co-occurring diseases. Multimorbidity is more complicated than just a sum of diseases, and the need to understand its patterns is well recognized [13]. The impacts of multimorbidity on CKD patients may vary according to the groups of diseases that occur together [14]. Recent research has identified some determinants of multimorbidity patterns, such as age and sex [15], but no study has directly addressed the association between these patterns and low health literacy. A better understanding of these associations may help to optimize care for CKD patients with low health literacy.

This study aims to assess the association between health literacy and the prevalence and patterns of multimorbidity in CKD patients, overall and divided by age and sex.

\section{Methods}

\section{Sample and procedures}

The sample for this cross-sectional study was derived from the baseline assessment of the Lifelines study. Lifelines is a multi-disciplinary prospective population-based cohort study which examines, in a unique three-generation design, the health and health-related behaviors of 167,729 people living in the north of the Netherlands. It employs a broad range of investigative procedures to assess the biomedical, socio-demographic, behavioral, physical, and psychological factors which contribute to the health and disease of the general population, with a special focus on multimorbidity and complex genetics.

Baseline data collection for adults was performed between November 2006 and December $2013(n=152,737)$. Prior to their first visit to the Lifelines outpatient clinic, all participants were asked to complete a self-administered questionnaire, requiring them to provide information pertaining to their medical history, current diseases, use of medication, and health behaviors. Participants were also instructed to bring in a detailed list with information about their latest used medications. During their first visit to the clinic, all participants underwent clinical examination, followed by blood and urine collection, electrocardiography, and spirometry. In addition, current psychiatric disorders (depressive disorder and anxiety disorders) were assessed with a brief standardized diagnostic interview: The Mini International Neuropsychiatric Interview (MINI) 5.0.0. Afterward, participants answered the health literacy questionnaire. More detailed information about recruitment and data collection can be found elsewhere [16]. The Lifelines Cohort Study was conducted in accordance with the principles of the Declaration of Helsinki and the research code of the University Medical Center Groningen [16].
Participants were eligible for our study if they were 18 or older, had completed the health literacy questionnaire, and could be classified as having CKD based on their albuminuria and serum creatinine levels. Exclusion criteria for our study included pregnancy and a history of kidney transplantation, due to the changes in renal function and prognosis that these conditions entail. The eligible sample comprised 2,742 CKD patients, with a mean follow-up time of 4.2 years (standard deviation 1.2 years).

\section{Measurements}

\section{Health literacy}

Health literacy was measured using self-reported answers to the three validated questions from Chew et al. [17].

(1) How often do you have trouble understanding your medical situation because you have difficulty with the written information?

(2) How sure are you of yourself when you fill out medical forms?

(3) How often does someone help you with reading information materials from the hospital or another healthcare provider?

Participants answered these questions on a Likert scale ranging from 1 (Never/not at all) to 5 (Always/Very). We reversed the scores of the first and third questions and then added up the scores of all questions, resulting in a health literacy scale ranging from 3 to 15 . This was dichotomized as low (3-12) versus adequate health literacy (13 and above). This cut-off point was also used in previous studies using the same database, and leads to percentages of low and adequate health literacy comparable to those found in large-scale health literacy surveys in the Netherlands [18].

\section{Definition of chronic kidney disease (CKD)}

In accordance with the latest guidelines [19], to be classified as having CKD a participant needed to meet at least one of the following criteria:

(1) Estimated glomerular filtration rate (eGFR) lower than $60 \mathrm{ml} / \mathrm{min} / 1.73 \mathrm{~m}^{2}$;

(2) Albuminuria assessed by $24 \mathrm{~h}$ urine $\geq 30 \mathrm{mg} / 24 \mathrm{~h}$ or assessed by albumin-to-creatinine ratio in morning urine $\geq 3 \mathrm{mg} / \mathrm{mmol}$.

The eGFR was calculated with the CKD-EPI formula, which uses values of serum creatinine, age, and sex. Creatinine levels were assessed with serum laboratory tests performed on fasting blood samples drawn from participants 
at one of the Lifelines research sites. On the same day, participants had to hand in urine samples collected at home. In the sample of adults who completed the health literacy questionnaire, 54\% had no values of albuminuria, and only their eGFR was used in the definition of CKD.

\section{Comorbidities and the definition of multimorbidity}

Forty comorbidities, i.e., single chronic diseases accompanying CKD, were scored according to the 10th edition of the International Statistical Classification of Diseases and Related Health Problems (ICD-10), using a combination of data from the questionnaires, medication list, clinical examination, laboratory assessment, electrocardiography, and spirometry. The combination of objective and subjective methods helped circumvent disease misclassification. Individuals had to meet at least one of the diagnosis conditions to be classified as affected by the corresponding comorbidity (for a detailed list of the classification criteria, see Supplementary Appendix I). The comorbidities were then clustered into 11 different disease domains, as previously reported [20]: gastrointestinal, cardiovascular, endocrine, pulmonary, central nervous system, ophthalmic and otorhinolaryngologic, psychiatric, musculoskeletal, hematologic, genitourinary, and dermatologic. A disease domain was considered as affected when at least one comorbidity was present within that disease domain. This clustering was made because individuals within a domain are likely to be treated by professionals of the same medical specialty, and with similar medications, therefore bearing a similar burden. Cancer was not included because different types of cancer have different physiopathology, risk factors, treatments, prognosis, and could be part of any of the aforementioned disease domains. Therefore, the use of cancer as overall diagnosis would hinder the precision of our comorbidity classification. The disease domains were posteriorly categorized into five groups: $0,1,2,3$, and 4 or more disease domains (in addition to CKD). Multimorbidity was present when, simultaneous with $\mathrm{CKD}$, at least one other disease domain was affected.

\section{Other variables}

In a questionnaire, participants reported sex, age, smoking, educational level, and monthly household income. We calculated BMI using height and weight measured by trained technicians at one of the Lifelines research sites. We defined participants as smokers if they reported any smoking in the previous month $[\mathrm{y} / \mathrm{n}]$. We measured educational level using an 8-item ordinal scale (from No education to University education), and subsequently categorized the answers as: low (no education or complete primary education), intermediate (complete secondary education), and high (higher vocational or university education). We measured monthly household income using an 8-item ordinal scale (from less than 750 euros to more than 3500 euros), and I do not know or I would rather not answer this question. The answers were clustered into four categories: less than 1000 euros, 1000 to 3000 euros, more than 3000 euros, and information not given.

\section{Analysis}

First, we calculated descriptive statistics and evaluated differences between the low- and adequate-health-literacy groups using Pearson's chi-square tests, independent samples t-tests, or Mann-Whitney tests. Second, we assessed the association between health literacy and the categorized number of disease domains, using multinomial logistic regression. The regression was adjusted for age, sex, eGFR, smoking, and BMI. Age was centralized and included as a confounder with its quadratic polynomial, to allow optimal fitting to the data. The eGFR was dichotomized into higher or lower than $60 \mathrm{ml} / \mathrm{min} / 1.73 \mathrm{~m}^{2}$. The results of all the models were considered statistically significant if $\mathrm{p}<0.05$. Results referring to groups with fewer than ten individuals were reported as $<10$ (or equivalent percentage), in accordance with the Lifelines requirements for publication to avoid the identification of participants. Subgroup analyses per CKD stage were not possible due to the small power yielded by the subsamples. Third, we assessed differences in patterns of multimorbidity by health literacy, using latent class analysis to define groups of disease domains. Latent class analysis is a model-based approach used to identify homogeneous groups within a heterogeneous population. Individuals in the same class share the same disease probability profile. We examined models with two to eight classes and selected the final model based on the lowest Bayesian information criterion (BIC), the lowest Akaike information criterion (AIC), and the clinical interpretability of the resulting classes. We performed this analysis separately for participants with low and adequate health literacy in the total sample. All analyses were then stratified by sex and age $(<65$ and $>=65$ ), to account for the known association of these variables with health literacy and multimorbidity [21, 22]. The analyses were conducted using IBM SPSS Statistics version 25, Stata version 16, and $R$ version 3.5.2 (package poLCA) for Windows. 
Table 1 Comparison of baseline characteristics between CKD patients with low and adequate health literacy

\begin{tabular}{|c|c|c|c|c|}
\hline Variables & $\begin{array}{l}\text { Adequate health } \\
\text { literacy }(\mathrm{n}=1816)\end{array}$ & $\begin{array}{l}\text { Low health } \\
\text { literacy }(n=926)\end{array}$ & $P$ value & $\mathrm{n}$ \\
\hline \multicolumn{5}{|c|}{ Demographic characteristics } \\
\hline Sex $\%$ of females & 54.6 & 57.9 & $0.10^{\mathrm{a}}$ & 2742 \\
\hline Age mean, $(S D)$ & $52.7(15.7)$ & $58.0(15.4)$ & $<0.001^{\text {b }}$ & 2742 \\
\hline \multicolumn{5}{|l|}{ Education \% } \\
\hline Low & 3.2 & 11.8 & $<\mathbf{0 . 0 0 1}^{\mathrm{a}}$ & 2638 \\
\hline Intermediate & 63.6 & 77.4 & & \\
\hline High & 33.1 & 10.9 & & \\
\hline \multicolumn{5}{|c|}{ Monthly household income \% } \\
\hline$<1000 €$ & 5.8 & 6.8 & $<0.001^{\mathrm{a}}$ & 2652 \\
\hline $1000-3000 €$ & 54.1 & 60.2 & & \\
\hline$>3000 €$ & 26.5 & 15.2 & & \\
\hline Information not given & 13.7 & 17.8 & & \\
\hline \multicolumn{5}{|l|}{ Clinical characteristics } \\
\hline Smoking $\%$ & 18.2 & 19.8 & $0.30^{\mathrm{a}}$ & 2716 \\
\hline BMI median, $(I Q R)$ & $26.5(5.9)$ & $27.4(5.4)$ & $<0.001^{\mathrm{c}}$ & 2741 \\
\hline \multicolumn{5}{|c|}{ Number of disease domains* $\%$} \\
\hline 0 & 14.4 & 7.9 & $<\mathbf{0 . 0 0 1}^{\mathrm{a}}$ & 2742 \\
\hline 1 & 21.6 & 14.8 & & \\
\hline 2 & 23.4 & 21.9 & & \\
\hline 3 & 21.2 & 26.6 & & \\
\hline 4 or more & 19.4 & 28.8 & & \\
\hline eGFR median, $(I Q R)$ & $80.4(42.0)$ & $69.8(40.6)$ & $<0.001^{\mathrm{c}}$ & 2742 \\
\hline \multicolumn{5}{|l|}{ CKD stage $\%$} \\
\hline 1 & 38.8 & 31.4 & $<\mathbf{0 . 0 0 1}^{\mathrm{a}}$ & 2742 \\
\hline 2 & 22.9 & 22.9 & & \\
\hline 3 & 37.4 & 44.4 & & \\
\hline 4 and 5 & 0.9 & 1.3 & & \\
\hline
\end{tabular}

$C K D$ Chronic kidney disease, $B M I$ body mass index, $e G F R$ estimated glomerular filtration rate $\mathrm{P}$ values $<0.05$ marked in bold

*Comorbidities grouped into 11 disease domains according to organ system

${ }^{a}$ Method: Pearson's chi-square test

${ }^{\mathrm{b}}$ Method: independent samples $t$ test

${ }^{c}$ Method: Mann-Whitney test

\section{Results}

\section{Sample characteristics}

Multimorbidity was present in $57 \%$ of the total Lifelines sample and $83.3 \%$ of the CKD patients. In the total Lifelines sample, $2.8 \%$ had CKD and $65 \%$ answered the health literacy questionnaire. Individuals who answered the questionnaire had fewer comorbidities. Participants with low health literacy were more likely to be older, and have a low socioeconomic status and higher BMI (Table 1).

\section{Association between low health literacy and multimorbidity}

Patients with low health literacy had more diseases simultaneously with their CKD (Table 2 and Fig. 1). This trend remained the same after stratification by sex and age, and older patients presented with more diseases than younger ones (Supplementary Appendix II and III). The number of diseases presented by one patient ranged from 0 to 14 , and the number of disease domains from 0 to 8 . Having low health literacy increased the chance of 
Table 2 Comparison of baseline prevalence of single diseases and disease domains between CKD patients with low and adequate health literacy

\begin{tabular}{|c|c|c|c|c|}
\hline Diseases & $\begin{array}{l}\text { Total sample } \\
(\mathrm{n}=2742)^{\mathrm{a}}\end{array}$ & $\begin{array}{l}\text { Adequate health } \\
\text { literacy }(\mathrm{n}=1816)\end{array}$ & $\begin{array}{l}\text { Low health literacy } \\
(\mathrm{n}=926)\end{array}$ & $P$ value ${ }^{b}$ \\
\hline Gastrointestinal diseases \% & 61.3 & 57.8 & 68.4 & $<0.001$ \\
\hline Fatty liver disease $\%$ & 60.3 & 56.8 & 67.2 & $<0.001$ \\
\hline Gastric disease \% & 2.9 & 2.0 & 4.5 & $<0.001$ \\
\hline Ulcerative colitis $\%$ & 0.8 & 0.9 & $<1.1$ & 0.33 \\
\hline Crohn's disease \% & $<0.4$ & $<0.6$ & $<1.1$ & 0.38 \\
\hline Celiac disease $\%$ & $<0.4$ & $<0.6$ & $<1.1$ & 0.63 \\
\hline Cardiovascular diseases \% & 44.2 & 41.0 & 50.4 & $<0.001$ \\
\hline Hypertension \% & 43.2 & 40.0 & 49.5 & $<0.001$ \\
\hline Heart failure $\%$ & 8.9 & 8.0 & 10.6 & 0.02 \\
\hline Vascular disease \% & 7.9 & 7.2 & 9.4 & 0.04 \\
\hline Atrial fibrillation $\%$ & 3.4 & 3.0 & 4.3 & 0.07 \\
\hline Pacemaker \% & 0.8 & 0.7 & 1.1 & 0.32 \\
\hline Heart transplant \% & $<0.4$ & $<0.6$ & $<1.1$ & 0.99 \\
\hline Endocrine diseases \% & 38.6 & 34.3 & 47.1 & $<0.001$ \\
\hline Hypercholesterolemia \% & 31.8 & 28.5 & 38.3 & $<0.001$ \\
\hline Diabetes \% & 11.0 & 9.7 & 13.6 & 0.002 \\
\hline Hypothyroidism \% & $<0.4$ & $<0.6$ & $<1.1$ & 0.60 \\
\hline Hyperthyroidism \% & 0.4 & $<0.6$ & $<1.1$ & 0.56 \\
\hline Pulmonary diseases $\%$ & 33.2 & 31.4 & 36.5 & 0.008 \\
\hline COPD \% & 28.2 & 26.7 & 31.1 & 0.02 \\
\hline Asthma \% & 8.2 & 7.7 & 9.3 & 0.16 \\
\hline Central nervous system diseases $\%$ & 23.6 & 22.9 & 25.1 & 0.20 \\
\hline Migraine $\%$ & 17.0 & 17.2 & 16.5 & 0.66 \\
\hline Back or neck hernia $\%$ & 7.5 & 6.7 & 9.2 & 0.02 \\
\hline Epilepsy \% & 0.6 & $<0.6$ & 1.1 & 0.01 \\
\hline Multiple sclerosis \% & $<0.4$ & $<0.6$ & $<1.1$ & 0.77 \\
\hline $\begin{array}{l}\text { Ophthalmic and } \\
\text { Otorhinolaryngologic diseases \% }\end{array}$ & 10.8 & 9.3 & 13.8 & $<0.001$ \\
\hline Ophthalmic disease \% & 8.8 & 7.4 & 11.4 & $<0.001$ \\
\hline $\begin{array}{l}\text { Otorhinolaryngologic } \\
\text { disease } \%\end{array}$ & 2.5 & 2.1 & 3.1 & 0.12 \\
\hline Psychiatric diseases \% & 10.6 & 9.0 & 13.7 & $<0.001$ \\
\hline Anxiety disorder \% & 8.9 & 7.6 & 11.6 & 0.001 \\
\hline Depressive disorder $\%$ & 3.5 & 2.4 & 5.5 & $<0.001$ \\
\hline Musculoskeletal diseases \% & 6.5 & 5.7 & 8.2 & 0.01 \\
\hline Rheumatoid arthritis \% & 3.3 & 2.9 & 4.1 & 0.08 \\
\hline Arthrosis \% & 2.3 & 1.8 & 3.2 & 0.01 \\
\hline Gout $\%$ & 1.9 & 1.9 & 1.8 & 0.95 \\
\hline Osteoporosis \% & $<0.4$ & $<0.6$ & $<1.1$ & 0.09 \\
\hline Hematologic diseases \% & 3.0 & 3.0 & 3.0 & 0.94 \\
\hline Anemia \% & 1.3 & 1.3 & 1.4 & 0.77 \\
\hline Thrombotic disease $\%$ & 1.6 & 1.7 & 1.6 & 0.95 \\
\hline Hemorrhagic disease $\%$ & $<0.4$ & $<0.6$ & $<1.1$ & 0.63 \\
\hline Genitourinary diseases \% & 2.5 & 2.3 & 3.0 & 0.23 \\
\hline $\begin{array}{l}\text { Benign prostatic } \\
\text { hypertrophy } \%\end{array}$ & 2.2 & 1.9 & 2.7 & 0.16 \\
\hline Chronic bladder infection $\%$ & $<0.4$ & $<0.6$ & $<1.1$ & 0.28 \\
\hline Double ovary extirpation \% & $<0.4$ & $<0.6$ & $<1.1$ & 0.23 \\
\hline Dermatologic diseases \% & 2.4 & 2.5 & 2.1 & 0.43 \\
\hline Eczema \% & 1.9 & 1.9 & 1.7 & 0.71 \\
\hline Psoriasis \% & 0.4 & $<0.6$ & $<1.1$ & 0.52 \\
\hline Severe acne $\%$ & $<0.4$ & $<0.6$ & $<1.1$ & 0.31 \\
\hline
\end{tabular}

Dementia and Parkinson's disease not displayed: presented by fewer than $0.05 \%$ of patients

$P$ values $<0.05$ marked in bold 
Table 2 (continued)
COPD Chronic obstructive pulmonary disease. Participants considered 'affected' in a disease domain (in bold) if positive for any disease listed below corresponding domain

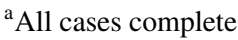

${ }^{\mathrm{b}}$ Method: Pearson's chi-square test

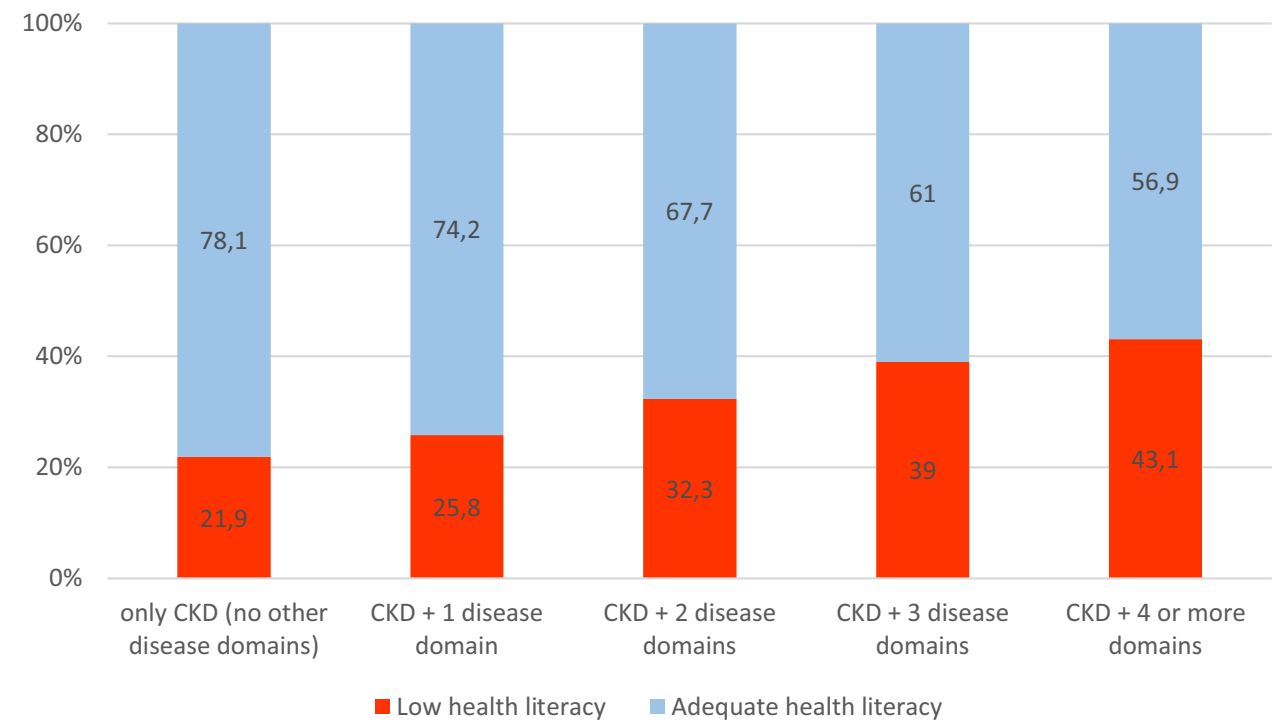

Fig. 1 Share of CKD patients with low and adequate health literacy by number of disease domains having multiple disease domains, and this association was stronger for greater numbers of disease domains (Table 3 ).

\section{Multimorbidity patterns in CKD patients with low and adequate health literacy}

The patterns of multimorbidity were very similar for both health literacy groups (Fig. 2, charts 1A and 1B). For both groups, the best-fitting model had four classes: (1) gastrointestinal and endocrine; (2) gastrointestinal, endocrine, cardiovascular, and pulmonary; (3) gastrointestinal, pulmonary, psychiatric, and neurologic; and (4) gastrointestinal. Supplementary Appendix IV shows the percentages of each disease domain per class. In the group with low health literacy, classes $1-4$ represented $2.6 \%, 21.4 \%, 16.4 \%$, and $59.6 \%$ of the sample, respectively. For the adequate health literacy groups, percentages were $3.1 \%, 14.3 \%, 13.4 \%$, and $69.2 \%$. Patterns were similar between health literacy groups across sex (Supplementary Appendix V). Patterns were also similar between health literacy groups among younger patients, but slightly different among older ones (Fig. 2, charts 2A-2D). Older patients with low health literacy had a relatively greater share of cardiovascular disease in class 4 , and psychiatric and central nervous system diseases in class 2 , when compared to older patients with adequate health literacy.

\section{Discussion}

This study showed that CKD patients have a high prevalence of comorbidities. Patients with low health literacy, especially when older, were more likely to have an even higher number of comorbidities. Moreover, for both levels of health literacy, the patterns of multimorbidity were similar, in the total sample and by sex. For the subgroup of older patients, however, those with low health literacy had a higher prevalence of most comorbidities, with a relatively greater share of cardiovascular, psychiatric, and central nervous system diseases.

Low health literacy is a strong independent indicator of the number of comorbidities among CKD patients. The association between low health literacy and comorbidities showed a dose-response gradient and was independent of age, sex, lifestyle, and CKD severity. This finding reinforces the robustness of the results, and the association most likely stems from the less effective health behaviors and self-management skills of patients with low health literacy [2]. These patients, thus, develop more comorbidities, which may negatively impact the prognosis of CKD. Our results contribute to the body of literature on the association between low health literacy and multimorbidity, which has until now shown mixed results [23,24]. Our study underlines the negative effects of low health literacy on multimorbidity, with a 


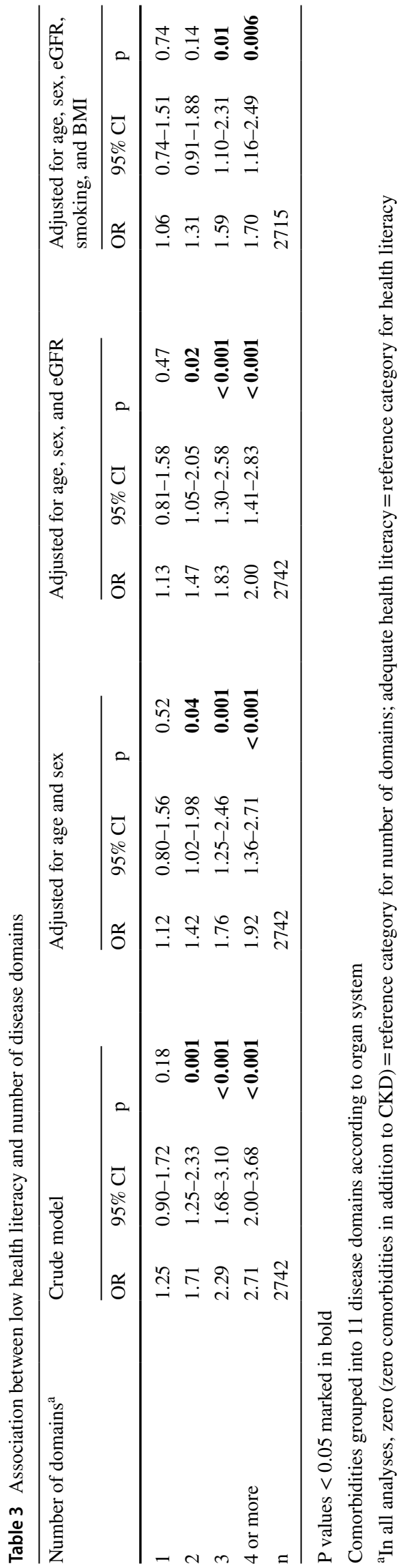

much larger sample and a more comprehensive set of comorbidities than in previous studies.

Interestingly, the patterns of multimorbidity were similar between patients with low and adequate health literacy in the total sample and when divided by sex, and only slightly different in older patients. These similar patterns indicate that, even though patients with low health literacy have more comorbidities than their adequate health literacy counterparts, the combination of comorbidities is roughly the same. This means that support of multimorbid CKD patients with low health literacy need not focus on different groups of diseases, but rather on general measures related to CKD care.

Among older adults, patients with low health literacy showed a higher prevalence of most comorbidities, which could be explained in two ways. First, patients with low health literacy have worse health behaviors and self-management skills, leading to aggravation of existing diseases and the development of new comorbidities, which accumulate over the life course [2]. A second explanation is that patients with low health literacy have poor access to care [25]. This could be due to a lack of resources to access care, or to a lack of knowledge to adequately use the available care. In either case, the result is a suboptimal use of medical resources for treating existing conditions and preventing new ones. The apparent differences in associations with health literacy by age suggest that the negative effects of low health literacy develop over time, being less in younger patients but more evident among older ones.

We found that patients with CKD had a high prevalence of specific comorbidities, which could overburden patients and negatively impact health outcomes. In the multimorbidity patterns studied, the most prevalent disease domains regarded gastrointestinal, endocrine, and cardiovascular diseases. These diseases are already known for co-occurring with CKD, given that they share various risk factors [26, 27]. The presence of multiple comorbidities increases the overall disease burden for patients [28]. Associated with this, it may lead to more polypharmacy with consequent poorer medication adherence, to increased psychological distress, and to a worse quality of life [1,29]. Furthermore, the presence of these comorbidities could accelerate eGFR decrease, either because combining different treatments becomes more intricate for patients, or because gastrointestinal, endocrine, and cardiovascular disease might be in the causal pathway toward CKD $[1,19,30]$.

Strengths of this study include its large population-based sample, with a wide selection of physical and mental chronic diseases, diagnosed by a combination of subjective and objective methods, using an internationally accepted coding system, and reported by age and sex. Furthermore, we employed latent class analysis, a sophisticated analysis technique to capture the heterogeneity in disease distribution. Latent class analysis is a person- rather than disease-oriented 
1A. Low health literacy (total sample)

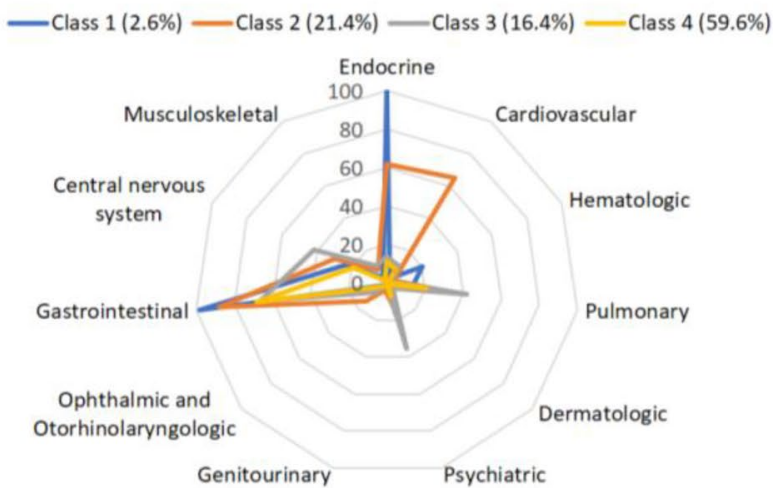

2A. Low health literacy (younger adults)

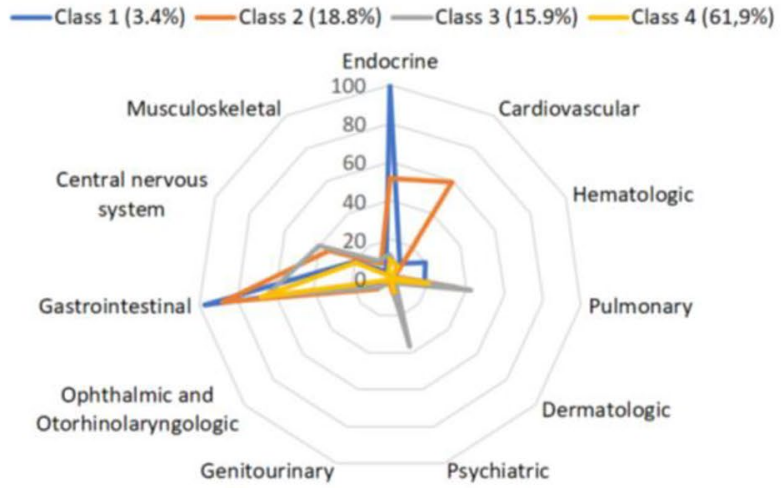

2C. Low health literacy (older adults)

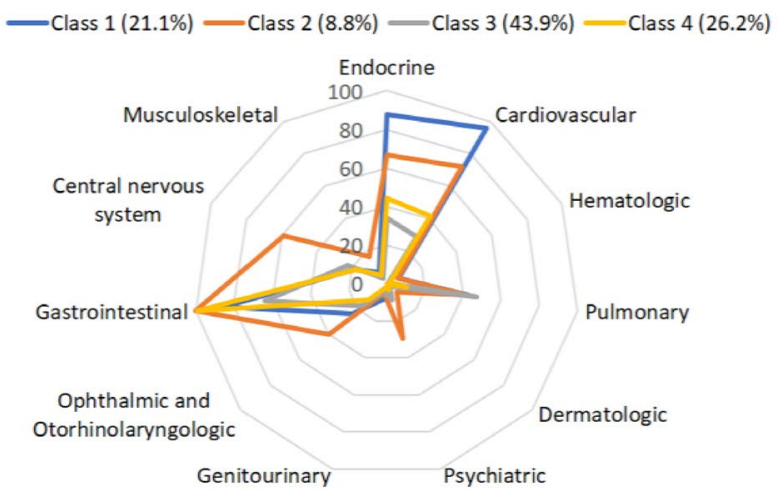

1B. Adequate health literacy (total sample)

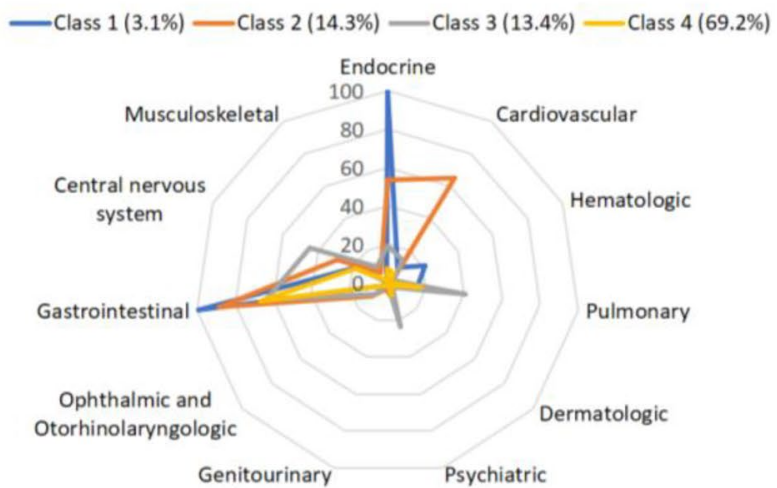

2B. Adequate health literacy (younger adults)

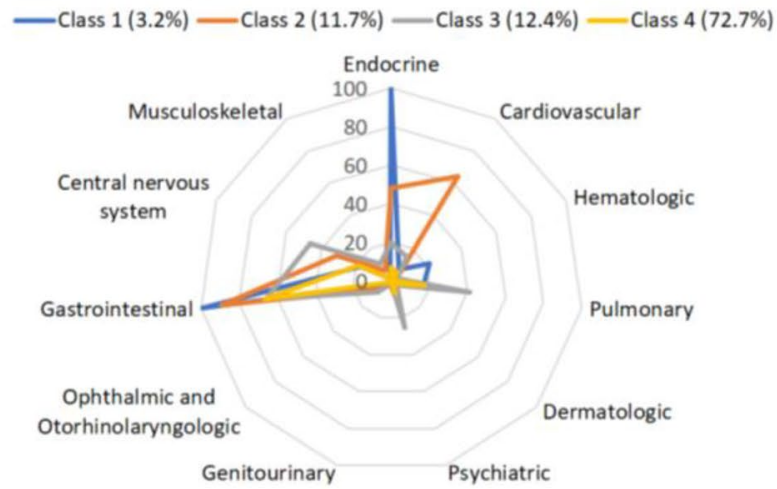

2D. Adequate health literacy (older adults)

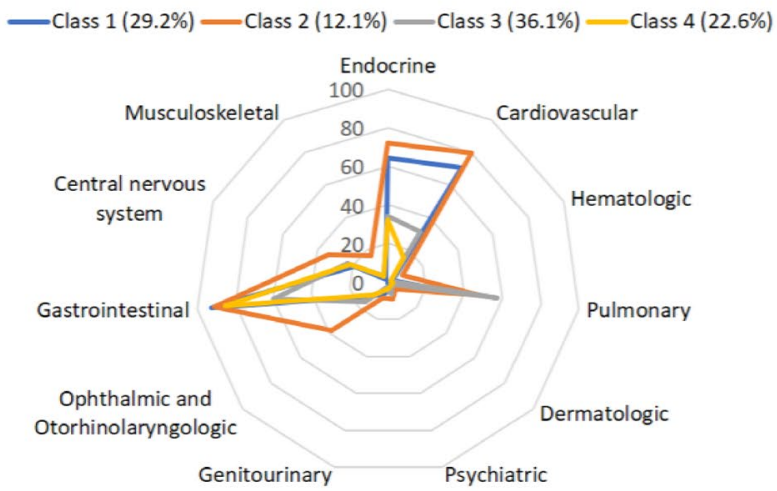

Fig. 2 Patterns of multimorbidity for groups of low and adequate health literacy, in total sample (1A and 1B) and stratified by age (2A-2D)

technique, providing results that meet current guidelines for patient-centered research and care.

Some limitations of our study should also be taken into account. First, we performed a cross-sectional analysis, which did not allow us to study whether multimorbidity patterns and their association with low health literacy change over time. Second, the health literacy questionnaire which we used is a self-report instrument focusing mainly on functional health literacy. This may have led to underestimations of low health literacy due to self-report, thereby reducing the power of our analysis. Nonetheless, our instrument is a validated tool that has been used in other studies [31]. Third, data on albuminuria were missing for $54 \%$ of our sample. However, this is unlikely to lead to important selection bias 
because albuminuria was assessed for a random subgroup of the Lifelines sample and its assessment stopped because of logistical reasons not related to CKD. Fourth, $65 \%$ of the participants answered the health literacy questionnaire. This reduced the power of our study, as the number of comorbidities was lower than among the participants that did not answer the questionnaire.

Public health practitioners and healthcare professionals should be aware of the importance of addressing low health literacy already at younger ages to prevent the development of multimorbidity during the life course. This aligns with current guidelines that focus more on prevention in healthcare services [32]. Moreover, the similar patterns of multimorbidity presented in both health literacy groups suggest that it is not necessary to change the focus of the care of low-health-literate patients regarding which groups of comorbidities should be prioritized. Care for them should be directed at the same comorbidities as those of their adequate-health-literate counterparts, but with extra support to overcome the challenges intrinsic to low health literacy. In clinical practice, this support could be achieved by using health-literacy-friendly strategies to improve self-management, health behaviors, and the quality and accessibility of CKD care. Patients could benefit from informational materials designed as narratives, such as photo stories, which are more recognizable, relevant, and engaging to patients [33, 34]. These materials could be used to promote the acquisition of health-related skills and CKD knowledge, and to increase patients' self-efficacy and motivation. Moreover, strengthening social support by engaging family members or friends of the patient could enhance disease management at home. Clinicians could also be supported by strategies to facilitate decision-making with patients with low health literacy [35]. This could be achieved through training to help clinicians tailor their communication strategies to the specific needs of each patient.

Future research should study the factors that mediate the association between low health literacy and multimorbidity to support the development of interventions to assist CKD patients. Ideally, research should be performed longitudinally, noting the trajectory and evolution of comorbidities, as well as health literacy, along the life course.

\section{Conclusion}

This study shows that, in a context of high prevalence of comorbidity, CKD patients with low health literacy are more likely to have a higher number of comorbidities than patients with adequate health literacy. Moreover, the multimorbidity patterns are similar for both groups of health literacy, differing slightly at older ages. This age difference suggests that the negative effects of low health literacy are more evident in aging groups. Therefore, improving low health literacy could be an intervention targeted to decrease multimorbidity along the life course of CKD patients.

Supplementary Information The online version contains supplementary material available at https://doi.org/10.1007/s40620-021-01229-1.

Acknowledgements The Lifelines Biobank initiative has been made possible by subsidy from the Dutch Ministry of Health, Welfare and Sport, the Dutch Ministry of Economic Affairs, the University Medical Center Groningen (UMCG the Netherlands), the University of Groningen, and the Northern Provinces of the Netherlands. The authors wish to acknowledge the services of the Lifelines Cohort Study, the contributing research centers delivering data to Lifelines, and all study participants.

Author contributions Conceptualization: MGdA, SR, GN, AdW; Methodology: all authors; Formal analysis and investigation: MGdA, JA; Writing — original draft preparation: MGdA; Writing—review and editing: SR, LM, JA, AdW; Project administration: MGdA; Supervision: SR, GN, AdW.

Funding None to declare.

Data availability The datasets analyzed during the current study are available in the Lifelines Biobank repository, at https://www.lifelines. nl/researcher.

Code availability Not applicable.

\section{Declarations}

Conflict of interest The authors have no relevant financial or non-financial interests to disclose.

Ethical approval The Lifelines Cohort Study was conducted in accordance with the principles of the Declaration of Helsinki and the research code of the University Medical Center Groningen.

Consent to participate Informed consent was obtained from all individual participants included in the Lifelines study.

Consent for publication Not applicable.

Open Access This article is licensed under a Creative Commons Attribution 4.0 International License, which permits use, sharing, adaptation, distribution and reproduction in any medium or format, as long as you give appropriate credit to the original author(s) and the source, provide a link to the Creative Commons licence, and indicate if changes were made. The images or other third party material in this article are included in the article's Creative Commons licence, unless indicated otherwise in a credit line to the material. If material is not included in the article's Creative Commons licence and your intended use is not permitted by statutory regulation or exceeds the permitted use, you will need to obtain permission directly from the copyright holder. To view a copy of this licence, visit http://creativecommons.org/licenses/by/4.0/. 


\section{References}

1. Fraser SD, Roderick PJ, May CR et al (2015) The burden of comorbidity in people with chronic kidney disease stage 3: a cohort study. BMC Nephrol 16:193

2. Mackey LM, Doody C, Werner EL, Fullen B (2016) Self-management skills in chronic disease management: what role does health literacy have? Med Decis Making 36:741-759

3. Navickas R, Petric V, Feigl AB, Seychell M (2016) Multimorbidity: what do we know? what should we do? J Comorb 6:4-11

4. Fraser SD, Taal MW (2016) Multimorbidity in people with chronic kidney disease: implications for outcomes and treatment. Curr Opin Nephrol Hypertens 25:465-472

5. Mucsi I, Kovacs AZ, Molnar MZ, Novak M (2008) Co-morbidity and quality of life in chronic kidney disease patients. J Nephrol 21:S84

6. Wallace E, Salisbury C, Guthrie B, Lewis C, Fahey T, Smith SM (2015) Managing patients with multimorbidity in primary care. BMJ 350:h176

7. Guthrie B, Payne K, Alderson P, McMurdo ME, Mercer SW (2012) Adapting clinical guidelines to take account of multimorbidity. BMJ 345:e6341

8. Kindig DA, Panzer AM, Nielsen-Bohlman L (2004) Health literacy: a prescription to end confusion. National Academies Press, Washington DC

9. Gurgel do Amaral MS, Reijneveld SA, Geboers B, Navis GJ, Winter AF. (2021) Low health literacy is associated with the onset of CKD during the life course. J Am Soc Nephrol 32:1436-1443

10. Gurgel do Amaral M, Reijneveld SA, Almansa J, Navis G, de Winter AF (2021) Do uncontrolled hypertension, diabetes, dyslipidemia, and obesity mediate the relationship between health literacy and chronic kidney disease complications. Int J Environ Res Public Health 18:5235

11. Geboers B, de Winter AF, Spoorenberg SL, Wynia K, Reijneveld SA (2016) The association between health literacy and self-management abilities in adults aged 75 and older, and its moderators. Qual Life Res 25:2869-2877

12. Fox CS, Matsushita K, Woodward M et al (2012) Associations of kidney disease measures with mortality and end-stage renal disease in individuals with and without diabetes: a meta-analysis. Lancet 380:1662-1673

13. Taylor AW, Price K, Gill TK et al (2010) Multimorbidity not just an older persons issue. Results from an Australian biomedical study. BMC Public Health 10:718

14. Brilleman SL, Purdy S, Salisbury C, Windmeijer F, Gravelle H, Hollinghurst S (2013) Implications of comorbidity for primary care costs in the UK: A retrospective observational study. Br J Gen Pract 63:e274-e282

15. Schäfer I, Hansen H, Schön G et al (2012) The influence of age, gender and socio-economic status on multimorbidity patterns in primary care. First results from the Multicare Cohort Study. BMC Health Serv Res 12:89

16. Scholtens S, Smidt N, Swertz MA et al (2015) Cohort profile: LifeLines, a three-generation cohort study and biobank. Int J Epidemiol 44:1172-1180

17. Chew LD, Griffin JM, Partin MR et al (2008) Validation of screening questions for limited health literacy in a large VA outpatient population. J Gen Intern Med 23:561-566

18. Sørensen K, Pelikan JM, Röthlin F et al (2015) Health literacy in Europe: comparative results of the European health literacy survey (HLS-EU). Eur J Public Health 25:1053-1058

19. Levin A, Stevens PE, Bilous RW et al (2013) Kidney disease: improving global outcomes (KDIGO) CKD work group. KDIGO
2012 clinical practice guideline for the evaluation and management of chronic kidney disease. Kidney Int Suppl 3:1-150

20. Meems LM, de Borst MH, Postma DS et al (2015) Low levels of vitamin D are associated with multimorbidity: results from the LifeLines cohort study. Ann Med 47:474-481

21. Martin LT, Ruder T, Escarce JJ et al (2009) Developing predictive models of health literacy. J Gen Intern Med 24:1211

22. Violan C, Foguet-Boreu Q, Flores-Mateo G et al (2014) Prevalence, determinants and patterns of multimorbidity in primary care: a systematic review of observational studies. PLoS ONE 9:e102149

23. Hudon C, Fortin M, Poitras M, Almirall J (2012) The relationship between literacy and multimorbidity in a primary care setting. BMC Fam Pract 13:33

24. Puente-Maestu L, Calle M, Rodríguez-Hermosa JL et al (2016) Health literacy and health outcomes in chronic obstructive pulmonary disease. Respir Med 115:78-82

25. Scott TL, Gazmararian JA, Williams MV, Baker DW (2002) Health literacy and preventive health care use among medicare enrollees in a managed care organization. Med Care 40:395-404

26. Kanbay M, Bulbul MC, Copur S et al (2021) Therapeutic implications of shared mechanisms in non-alcoholic fatty liver disease and chronic kidney disease. J Nephrol 34:649-659

27. Ejerblad E, Fored CM, Lindblad P, Fryzek J, McLaughlin JK, Nyren O (2006) Obesity and risk for chronic renal failure. J Am Soc Nephrol 17:1695-1702

28. Rosbach M, Andersen JS (2017) Patient-experienced burden of treatment in patients with multimorbidity - a systematic review of qualitative data. PLoS ONE 12:e0179916

29. Fortin M, Lapointe L, Hudon C, Vanasse A, Ntetu AL, Maltais D (2004) Multimorbidity and quality of life in primary care: a systematic review. Health Qual Life Outcomes 2:1-12

30. Kaps L, Labenz C, Galle PR, Weinmann-Menke J, Kostev K, Schattenberg JM (2020) Non-alcoholic fatty liver disease increases the risk of incident chronic kidney disease. United Eur Gastroenterol J 8:942-948

31. Geboers B, de Winter AF, Luten KA, Jansen CJ, Reijneveld SA (2014) The association of health literacy with physical activity and nutritional behavior in older adults, and its social cognitive mediators. J Health Commun 19(Suppl 2):61-76

32. England NHS (2014) Five year forward review. https://www.engla nd.nhs.uk/wp-content/uploads/2014/10/5yfv-web.pdf. Accessed 15 Dec 2019

33. van't Koops Jagt R, Tan SL, Hoeks J et al (2019) Using photo stories to support doctor-patient communication: evaluating a communicative health literacy intervention for older adults. Int J Environ Res Public Health 16:3726

34. Koops van't Jagt, Ruth, Hoeks JCJ, Jansen CJM, de Winter AF, Reijneveld SA. (2016) Comprehensibility of health-related documents for older adults with different levels of health literacy: a systematic review. J Health Commun 21:159-177

35. Boonstra MD, Reijneveld SA, Foitzik EM, Westerhuis R, Navis G, de Winter AF (2020) How to tackle health literacy problems in chronic kidney disease patients? A systematic review to identify promising intervention targets and strategies. Nephrol Dial Transplant 1:15

Publisher's Note Springer Nature remains neutral with regard to jurisdictional claims in published maps and institutional affiliations. 